\title{
GROWTH HYPERSPACES OF PEANO CONTINUA ${ }^{1}$
}

\author{
BY
}

D. W. CURTIS

\begin{abstract}
For $X$ a nondegenerate Peano continuum, let $2^{X}$ be the hyperspace of all nonempty closed subsets of $X$, topologized with the Hausdorff metric. It is known that $2^{x}$ is homeomorphic to the Hilbert cube. A nonempty closed subspace $\mathcal{G}$ of $2^{X}$ is called a growth hyperspace provided it satisfies the following condition: if $A \in \mathcal{G}$, and $B \in 2^{X}$ such that $B \supset A$ and each component of $B$ meets $A$, then also $B \in \mathcal{G}$. The class of growth hyperspaces includes many previously considered subspaces of $2^{X}$. It is shown that if $X$ contains no free arcs, and $\mathcal{G}$ is a nontrivial growth hyperspace, then $\mathcal{G} \backslash\{X\}$ is a Hilbert cube manifold. A corollary characterizes those growth hyperspaces which are homeomorphic to the Hilbert cube. Analogous results are obtained for growth hyperspaces with respect to the hyperspace $\operatorname{cc}(X)$ of closed convex subsets of a convex $n$-cell $X$.
\end{abstract}

1. Introduction. Wojdyslawski [17] showed that for every Peano continuum $X$, the hyperspace $2^{X}$ of nonempty closed subsets of $X$, topologized by the Hausdorff metric, is an absolute retract (AR) for the class of metrizable spaces. Kelley [10] gave another proof of this result which applies as well to any nonempty closed subspace $\mathcal{G}$ of $2^{X}$ satisfying the following condition: if $A \in \mathcal{S}$ and $B \in 2^{X}$ such that $B \supset A$ and each component of $B$ meets $A$, then $B \in \mathcal{G}$. We call such a subspace $\mathcal{G}$ a growth hyperspace of $X$. Note that always $X \in \mathcal{G}$. Kelley's proof shows that every growth hyperspace of a Peano continuum is an AR.

More recently, it has been shown [6], [7], [12], [13] that the hyperspace $2^{X}$ of every nondegenerate Peano continuum $X$ is homeomorphic to the Hilbert cube $Q$. This result has also been obtained (with additional hypotheses, in some cases) for certain other growth hyperspaces of $X$. In particular, if $A$ is a proper closed subset of $X$, the hyperspaces $2_{A}^{X}=\left\{F \in 2^{X} \mid F \supset A\right\}$ and $2^{X}(A)=\left\{F \in 2^{X} \mid F \cap A \neq \varnothing\right\}$ are both homeomorphic to $Q$ [8]. And if $X$ contains no free arcs (i.e., admits no open imbedding of the line), the hyperspace $C(X)$ of nonempty subcontinua of $X$, as well as the hyperspaces

Received by the editors April 7, 1976 and, in revised form, February 2, 1977.

AMS (MOS) subject classifications (1970). Primary 54B20, 54B25, 54F25, 54F50, 54F65, 57A20.

Key words and phrases. Hyperspaces, hyperspaces of convex subsets, Peano continuum, Hilbert cube manifold, inverse sequence, near-homeomorphism, local dendron.

'Research supported in part by NSF Grant GP 44349 
$C_{A}(X)=C(X) \cap 2_{A}^{X}$ and $C(X ; A)=C(X) \cap 2^{X}(A)$, are homeomorphic to $Q$.

Our main objective in this paper is the appropriate extension of these results to the general class of growth hyperspaces of a Peano continuum. Of course not every nontrivial growth hyperspace is homeomorphic to $Q$ (although Edwards' characterization of AR's as $Q$-factors [4] shows that every growth hyperspace is a $Q$-factor). For example, $\mathcal{G}=2_{[-1,0]}^{[-1,1]} \cup 2_{[0,1]}^{[-1,1]}$ is the union of two Hilbert cubes intersecting in a point. Other examples and a more general result appear in $\$ 5$. The extension takes the following form: under some rather general conditions, the subspace $\mathcal{G} \backslash\{X\}$ of every nontrivial growth hyperspace $\mathcal{G}$ is a $[0,1)$-stable $Q$-manifold. As a corollary, we obtain a useful characterization of those growth hyperspaces which are homeomorphic to $Q$. The above result also has a converse: for every $[0,1)-$ stable $Q$-manifold $M$ and nondegenerate Peano continuum $X$, there is a growth hyperspace $\mathcal{G}$ of $X$ such that $\mathcal{S} \backslash\{X\}$ is homeomorphic to $M$.

For a given Peano continuum $X$, the class of all growth hyperspaces of $X$, when partially ordered by set inclusion, forms a complete lattice, with lower bound $\{X\}$ and upper bound $2^{X}$. For any subcollection $\left\{\mathcal{G}_{\alpha}\right\}$ of growth hyperspaces, we have $\inf \left\{\mathcal{G}_{\alpha}\right\}=\cap\left\{\mathcal{G}_{\alpha}\right\}$ and $\sup \left\{\mathcal{G}_{\alpha}\right\}=\operatorname{cl} \cup\left\{\mathcal{G}_{\alpha}\right\}$. Thus every closed subspace $\mathscr{Q}$ of $2^{X}$ generates a growth hyperspace $\mathcal{S}_{\mathscr{Q}}=\inf \{\mathcal{G} \mid \mathcal{G}$ $\supset \mathbb{Q}\}$, and in fact $\mathcal{G}_{\mathscr{Q}}=\left\{F \in 2^{X} \mid\right.$ for some $A \in \mathbb{Q}, F \supset A$ and each component of $F$ meets $A$ \}.

A growth hyperspace $\mathcal{G}$ which satisfies the following stronger condition is called an inclusion hyperspace: if $A \in \mathcal{G}$ and $B \in 2^{X}$ such that $B \supset A$, then $B \in \mathcal{G}$. Thus $2_{A}^{X}$ and $2^{X}(A)$ are inclusion hyperspaces. The class of inclusion hyperspaces is a complete sublattice of the lattice of growth hyperspaces.

Finally, we consider the analogue of growth hyperspaces in the setting of the hyperspace $\operatorname{cc}(X)$ of nonempty closed convex subsets of a convex $n$-cell $X$.

2. Convex metrics. Every Peano continuum $X$ admits a convex metric [1]; i.e., a metric $d$ such that for each pair of distinct points $x$ and $y$, there exists an $\operatorname{arc} \alpha$ in $X$ between $x$ and $y$ which is isometric to the closed interval $[0, d(x, y)]$. Such a metric $d$ (which we may assume to be bounded by 1) defines a natural contraction $\eta$ of the hyperspace $2^{X}$ to the element $\{X\}$ : simply set $\eta(F, t)=\{x \in X \mid d(x, F) \leqslant t\}$. Note that $\eta\left(\eta\left(F, t_{1}\right), t_{2}\right)=\eta\left(F, t_{1}\right.$ $+t_{2}$ ).

There are two fundamental properties of a growth hyperspace $\mathcal{S}$ in relation to the map $\eta$ :

(1) $\eta(\mathcal{S} \times I) \subset \mathcal{G}$;

(2) the function $2^{X} \rightarrow I$, defined by $F \rightarrow t_{F}$ where $t_{F}$ is the smallest value of $t$ for which $\eta(F, t) \in \mathcal{G}$, is continuous. Consequently, for each pair of growth 
hyperspaces $\mathcal{G}$ and $\mathcal{K}$ such that $\mathcal{G} \subset \mathcal{K}$, there is a natural strong deformation retraction $\eta(\mathcal{G} ; \mathcal{F C}): \mathcal{H C} \rightarrow \mathcal{G}$, defined by $H \rightarrow \eta\left(H, t_{H}\right)$, and for growth hyperspaces $\mathscr{F} \subset \mathcal{G} \subset \mathcal{H}$, we have $\eta(\mathscr{F} ; \mathcal{G}) \eta(\mathcal{G} ; \mathcal{H})=\eta(\mathscr{F} ; \mathcal{H})$. In particular, the existence of the retraction $\eta\left(\mathcal{G} ; 2^{X}\right): 2^{X} \rightarrow \mathcal{G}$ gives another proof that every growth hyperspace is an AR. Note that each retraction $\eta(\mathcal{G} ; \mathcal{F})$ has contractible point-inverses.

In what follows, $X$ will always denote a Peano continuum.

3. Growth hyperspaces of Peano continua with no free arcs. For $A \in 2^{X}$, define $G_{A}(X)=\left\{F \in 2^{X} \mid F \supset A\right.$ and each component of $F$ meets $\left.A\right\}$. Thus $G_{A}(X)$ is the smallest growth hyperspace of $X$ containing $A$. The finite union $\cup_{i=1}^{n} G_{A_{i}}(X)$ of such is the smallest growth hyperspace containing $\left\{A_{1}, \ldots, A_{n}\right\}$, and will be useful in the proof of our main result.

The proofs in [7] that $2^{X} \approx Q$ and, for $X$ containing no free arcs, that $C(X) \approx Q$, are based on the construction of inverse sequences of hyperspaces of local dendra imbedded in $X$. These local dendra are constructed as 1-dimensional nerves of partitions of $X$, and if $X$ contains no free arcs neither do the local dendra (i.e., the set of branch points is dense). In the following proofs we again consider such local dendra and certain of their growth hyperspaces. The local dendra themselves are most easily viewed as inverse limits of graphs. Thus, let $D=\operatorname{inv} \lim \left(\Gamma_{i}, r_{i}\right)$, where each $\Gamma_{i} \subset D$ is a compact connected graph and each bonding map $r_{i}: \Gamma_{i+1} \rightarrow \Gamma_{i}$ is a $\mathrm{PL}$ retraction with contractible point-inverses. Thus the projection map $r: D \rightarrow$ $\Gamma_{1}$ is a deformation retraction. We refer to $\Gamma_{1}$ as the base of the local dendron D.

Lemma 3.1. Let $A_{1}, \ldots, A_{n}$ be closed subsets of $D$ such that each $A_{i}=$ $r^{-1}\left(r\left(A_{i}\right)\right)$ and $\cup_{i=1}^{n} A_{i} \neq D$. Then if the set of branch points of $D$ is dense, the growth hyperspace $\cup_{i=1}^{n} G_{A_{i}}(D)$ is homeomorphic to $Q$.

Proof. Let $\mathscr{F}=\left\{F \in \cup_{i=1}^{n} G_{A_{i}}(D) \mid F \subset \cup\left\{A_{i} \mid F \in G_{A_{i}}(D)\right\} \cup \Gamma_{1}\right\}$. We show that $\mathscr{F}$ is a $Z$-set in $\cup_{i=1}^{n} G_{A_{i}}(D)$, and that $\cup_{i=1}^{n} G_{A_{i}}(D) \backslash \mathscr{F}$ is a $Q$-manifold. The lemma then follows from a result of Torunczyk [14]: if $Y$ is a $Q$-factor, $A \subset Y$ is a $Z$-set, and $Y \backslash A$ is a $Q$-manifold, then $Y$ is homeomorphic to $Q$.

For each $\varepsilon>0$ we must find a map $f: \bigcup_{i=1}^{n} G_{A_{i}}(D) \rightarrow \bigcup_{i=1}^{n} G_{A_{i}}(D) \backslash \mathscr{F}$ such that $\rho(f, \mathrm{id})<\varepsilon$. Here $\rho$ is the Hausdorff hyperspace metric induced by a convex metric $d$ on $D$. Using the fact that $D$ has a dense set of branch points, we may choose $0<\delta<\varepsilon / 2$ such that, for any subinterval $J$ of the base $\Gamma_{1}$ with length $\varepsilon / 4, \rho\left(J, r^{-1}(J)\right)>\delta$. Now define maps $f_{1}$ and $f_{2}$ of $\cup_{i=1}^{n} G_{A_{i}}(D)$ into itself as follows: $f_{1}(B)=B \cup\left\{x \in \Gamma_{1} \mid d\left(x, B \cap \Gamma_{1}\right)<\right.$ $\varepsilon / 2\}$ and $f_{2}(B)=\{x \in D \mid d(x, B) \leqslant \delta\}$. Then $\rho\left(f_{2} f_{1}\right.$, id $) \leqslant \varepsilon / 2+\delta<\varepsilon$, and we claim that $f=f_{2} f_{1}$ maps off of $\mathscr{F}$. Consider $B \in \cup_{i=1}^{n} G_{A_{i}}(D)$, and 
suppose first that $f_{1}(B) \cap \Gamma_{1}$ (which is simply the closed $\varepsilon / 2$-neighborhood of $B \cap \Gamma_{1}$ in $\left.\Gamma_{1}\right)$ is not contained in $\bigcup_{i=1}^{n} r\left(A_{i}\right)$. Then clearly $f_{2}\left(f_{1}(B)\right) \notin \mathscr{F}$. On the other hand, if $f_{1}(B) \cap \Gamma_{1} \subset \bigcup_{i=1}^{n} r\left(A_{i}\right)$, then since $\bigcup_{i=1}^{n} r\left(A_{i}\right) \neq \Gamma_{1}$ and $\Gamma_{1}$ is connected, $f_{1}(B) \cap \Gamma_{1}$ must contain a subinterval $J$ of $\Gamma_{1}$ with length $\varepsilon / 4$ such that $J \cap B=\varnothing$. Then by the choice of $\delta$, we again have $f_{2}\left(f_{1}(B)\right) \notin \mathscr{F}$. Thus $\mathscr{F}$ is a $Z$-set in $\bigcup_{i=1}^{n} G_{A_{i}}(D)$.

Now consider an element $B$ of $\cup_{i=1}^{n} G_{A_{i}}(D) \backslash \mathscr{F}$. Let $x \in B \backslash \Gamma_{1}$ such that $x \notin \cup\left\{A_{i} \mid B \in G_{A_{i}}(D)\right\}$, and let $x_{0}=r(x)$. Then $B$, and every element of $\cup_{i=1}^{n} G_{A_{i}}(D)$ sufficiently close to $B$, must contain $x_{0}$. Let $D_{0}=\left(D \backslash r^{-1}\left(x_{0}\right)\right)$ $\cup\left\{x_{0}\right\}$. Clearly, both $D_{0}$ and $r^{-1}\left(x_{0}\right)$ are compact connected local dendra whose sets of branch points are dense. Thus the growth hyperspace $G_{0}=$ $\cup\left\{G_{A_{i}}\left(D_{0}\right) \mid B \in G_{A_{i}}(D)\right\} \cap 2_{x_{0}}^{D_{0}}$ is a $Q$-factor, and the hyperspace of subcontinua $C_{x_{0}}\left(r^{-1}\left(x_{0}\right)\right)$ is homeomorphic to $Q$ [13]. Hence the product $G_{0} \times$ $C_{x_{0}}\left(r^{-1}\left(x_{0}\right)\right)$ is homeomorphic to $Q$, and clearly this product is homeomorphic to a neighborhood of the element $B$ in $\cup_{i=1}^{n} G_{A_{i}}(D)$. Therefore $\bigcup_{i=1}^{n} G_{A_{i}}(D) \backslash \mathscr{F}$ is a $Q$-manifold.

Lemma 3.2. Let $X$ be a Peano continuum with no free arcs, and $A_{1}, \ldots, A_{n}$ nonempty closed subsets of $X$ such that $\cup_{i=1}^{n} A_{i} \neq X$. Then for each $\varepsilon>0$ there exist elements $B_{1} \in G_{A_{1}}(X), \ldots, B_{n} \in G_{A_{n}}(X)$ with $B_{1} \subset \eta\left(A_{1}, \varepsilon\right), \ldots, B_{n} \subset$ $\eta\left(A_{n}, \varepsilon\right)$, such that $\cup_{i=1}^{n} G_{B_{i}}(X)$ is homeomorphic to $Q$.

Proof. We use the construction of partition refinements, nerves, and local dendra detailed in [7] for the proof that the hyperspace of subcontinua $C(X)$ is homeomorphic to $Q$. This construction yielded a sequence $\left\{G_{i}\right\}$ of partitions of $X$, with each $G_{i+1}$ refining $G_{i}$ and mesh $G_{i} \rightarrow 0$, a corresponding sequence $\left\{\Gamma_{i}\right\}$ of compact connected graphs in $X$, with each $\Gamma_{i}$ a nerve of $G_{i}$, and a sequence $\left\{D_{i}\right\}$ of compact connected local dendra in $X$, with each $D_{i}$ based on $\Gamma_{i}$ (see the discussion preceding Lemma 3.1) and having a dense set of branch points. For each partition element $g \in G_{i}$, we have $D_{i} \cap \operatorname{Bd} g=\Gamma_{i}$ $\cap \mathrm{Bd} g$ and $D_{i} \cap \bar{g}=p_{i}^{-1}\left(\Gamma_{i} \cap \bar{g}\right)$, where $p_{i}: D_{i} \rightarrow \Gamma_{i}$ is the projection map. Furthermore, there exists a sequence of $C$-monotone piecewise-linear maps $\varphi_{i}: \Gamma_{i+1} \rightarrow C\left(\Gamma_{i}\right)$, inducing in turn sequences of near-homeomorphisms $f_{i}$ : $2^{D_{i+1}} \rightarrow 2^{D_{i}}$ and $g_{i}=\left.f_{i}\right|_{C\left(D_{i+1}\right)}: C\left(D_{i+1}\right) \rightarrow C\left(D_{i}\right)$, such that

$$
C(X) \approx \operatorname{inv} \lim \left(C\left(D_{i}\right), g_{i}\right) \approx Q
$$

(using the inverse sequence Approximation Lemma 2.1 of [6]).

For each $i \leqslant n$, let $G_{1}\left(A_{i}\right)=\left\{g \in G_{1} \mid \bar{g} \cap A_{i} \neq \varnothing\right\}$ and $A_{i, 1}=\cup\{\bar{g} \mid g \in$ $\left.G_{1}\left(A_{i}\right)\right\}$. Then $A_{i} \subset$ int $A_{i, 1}, A_{i, 1} \in G_{A_{i}}(X)$, and if mesh $G_{1}$ is small enough, $A_{i, 1} \subset \eta\left(A_{i}, \varepsilon\right)$ and $\cup_{i=1}^{n} A_{i, 1} \neq X$. It follows from Lemma 3.1 that $\cup_{i=1}^{n} G_{A_{i, 1} \cap D_{1}}\left(D_{1}\right) \approx Q$

The construction in [7] of the partition refinement $G_{2}$, nerve $\Gamma_{2}$, and $C$-monotone map $\varphi_{1}: \Gamma_{2} \rightarrow C\left(\Gamma_{1}\right)$ is such that, for arbitrary $\delta>0$, we may 
assume that for each $g \in G_{2}, \varphi_{1}\left(\bar{g} \cap \Gamma_{2}\right) \subset \cup\left\{\bar{g}^{\prime} \mid g^{\prime} \in G_{1}\right.$ and $g \cap N_{\delta}\left(g^{\prime}\right)$ $\neq \varnothing\}$. Now set $G_{2}\left(A_{i}\right)=\left\{g \in G_{2} \mid g \subset g^{\prime} \in G_{1}\left(A_{i}\right)\right.$ and $\left.\varphi_{1}\left(\bar{g} \cap \Gamma_{2}\right) \subset A_{i, 1}\right\}$, and $A_{i, 2}=\cup\left\{\bar{g} \mid g \in G_{2}\left(A_{i}\right)\right\}$. It follows that if mesh $G_{2}$ is small enough, $A_{i} \subset$ int $A_{i, 2}$ and $A_{i, 2} \in G_{A_{i}}(X)$. Again by Lemma 3.1, $\cup_{i=1}^{n} G_{A_{i, 2} \cap D_{2}}\left(D_{2}\right) \approx Q$.

We claim that the map $f_{1}: 2^{D_{2}} \rightarrow 2^{D_{1}}$, induced essentially by the map $\varphi_{1}$, restricts to a surjection $f_{1}: \bigcup_{i=1}^{n} G_{A_{i, 2} \cap D_{2}}\left(D_{2}\right) \rightarrow \bigcup_{i=1}^{n} G_{A_{i, 1} \cap D_{1}}\left(D_{1}\right)$ with contractible point-inverses. First note that, because $\varphi_{1}$ is $C$-monotone, and due to the choice of $G_{2}\left(A_{i}\right)$, we have $f_{1}\left(A_{i, 2} \cap D_{2}\right)=A_{i, 1} \cap D_{1}$ for each $i \leqslant n$. Thus $f_{1}\left(\cup_{i=1}^{n} G_{A_{i, 2} \cap D_{2}}\left(D_{2}\right)\right) \subset \cup_{i=1}^{n} G_{A_{i, 1} \cap D_{1}}\left(D_{1}\right)$. The argument that each point-inverse of the restriction of $f_{1}$ is nonempty and contractible follows from the argument given for Theorem 3.5 of [6]. Thus, for arbitrary $K \in$ $G_{A_{i, 1} \cap D_{1}}\left(D_{1}\right), i \leqslant n$, set $K^{\prime}=\left\{x \in D_{2} \mid f_{1}(\{x\}) \subset K\right\}$. Then $f_{1}\left(K^{\prime}\right)=K$, and $K^{\prime} \in G_{A_{i, 2} \cap D_{2}}\left(D_{2}\right)$. Moreover, the "expansion homotopy" which contracts the point-inverse $f_{1}^{-1}(K) \subset 2^{D_{2}}$ of $f_{1}: 2^{D_{2}} \rightarrow 2^{D_{1}}$ to the element $K^{\prime}$ restricts to a contraction of $f_{1}^{-1}(K) \cap G_{A_{i, 2} \cap D_{2}}\left(D_{2}\right)$ to $K^{\prime}$, since $G_{A_{i, 2} \cap D_{2}}\left(D_{2}\right)$ is a growth hyperspace.

By a theorem of Chapman [4], every surjection between copies of $Q$ which has contractible point-inverses is a near-homeomorphism. Thus the above restriction of $f_{1}$ is a near-homeomorphism.

Continuing in this fashion, we inductively set $G_{k+1}\left(A_{i}\right)=\left\{g \in G_{k+1} \mid g \subset\right.$ $g^{\prime} \in G_{k}\left(A_{i}\right)$ and $\left.\varphi_{k}\left(\bar{g} \cap \Gamma_{k+1}\right) \subset A_{i, k}\right\}$, and $A_{i, k+1}=\bigcup\left\{\bar{g} \mid g \in G_{k+1}\left(A_{i}\right)\right\}$, for each $i \leqslant n$ and $k \geqslant 1$. If mesh $G_{k+1}$ is small enough, $A_{i, k+1} \in G_{A_{i}}(X)$. As before, the restricted map $f_{k}: \cup_{i=1}^{n} G_{A_{i, k+1} \cap D_{k+1}}\left(D_{k+1}\right) \rightarrow \bigcup_{i=1}^{n} G_{A_{i, k} \cap D_{k}}\left(D_{k}\right)$ is a near-homeomorphism. Then taking $B_{i}=\bigcap_{k=1}^{\infty} A_{i, k}$ for each $i$, we obtain

$$
\bigcup_{i=1}^{n} G_{B_{i}}(X) \approx \operatorname{inv} \lim \left(\bigcup_{i=1}^{n} G_{A_{i, k} \cap D_{k}}\left(D_{k}\right), f_{k}\right) \approx Q .
$$

A $Q$-manifold $M$ is [0,1)-stable if $M \approx M \times[0,1)$. Chapman [2] showed that $[0,1)$-stable $Q$-manifolds are topologically classified by homotopy type. Wong [18] showed that $M$ is $[0,1)$-stable if and only if $M$ is properly contractible to infinity (i.e., for every compact subset $K \subset M$, there exists a proper homotopy $\left\{f_{t}\right\}: M \rightarrow M$ with $f_{0}=$ id and $\left.f_{1}(M) \subset M \backslash K\right)$.

THEOREM 3.1. If $\mathcal{G}$ is a nontrivial growth hyperspace of a Peano continuum $X$ with no free arcs, then $\mathcal{G} \backslash\{X\}$ is a $[0,1)$-stable $Q$-manifold.

Proof. Let $A \in \mathcal{G} \backslash\{X\}$, and let $\left\{A_{i}\right\}$ be a sequence in $\mathcal{G}$ which is dense in a neighborhood of $A$ in $\mathcal{G}$, and such that $\cup_{1}^{\infty} A_{i} \neq X$. By Lemma 3.2 there exists, for each $j \geqslant 1$ and $0<\varepsilon_{j}<2^{-j}$, elements $B_{1}^{j}, \ldots, B_{j}^{j}$ of $\mathcal{G}$ such that each $B_{i}^{j} \in G_{A_{i}}(X), B_{i}^{j} \subset \eta\left(A_{i}, \varepsilon_{j}\right)$, and $\bigcup_{i=1}^{j} G_{B_{i}^{j}}(X) \approx Q$. We may assume also that each $B_{i}^{j}$ is a neighborhood of $A_{i}$ (simply replace each $A_{i}$ by a sufficiently small neighborhood $\eta\left(A_{i}, \delta\right)$ before applying Lemma 3.2). Thus 
the sequence of positive constants $\left\{\varepsilon_{j}\right\}$ may be inductively chosen so that $B_{i}^{j+1} \subset B_{i}^{j}$ for each $i \leqslant j$, with $\bigcap_{j=i}^{\infty} B_{i}^{j}=A_{i}$ for each $i$. Note that

for each $j$, and

$$
\bigcup_{i=1}^{j} G_{B_{i}^{j}}(X) \subset \bigcup_{i=1}^{j+1} G_{B_{i}^{j+1}}(X) \subset \bigcup_{i=1}^{j+1} G_{A_{i}}(X) \subset \mathcal{G}
$$

$$
\lim _{j \rightarrow \infty} \bigcup_{i=1}^{j} G_{B_{i}^{j}}(X)=\mathrm{cl} \bigcup_{j=1}^{\infty} \bigcup_{i=1}^{j} G_{B_{i}^{j}}(X) \supset \mathrm{cl} \bigcup_{i=1}^{\infty} G_{A_{i}}(X)
$$

is a neighborhood of $A$ in $\mathcal{G}$ (the limit operation indicated by $\lim _{j \rightarrow \infty}$ takes place in the space $2^{\mathcal{G}}$ of closed subspaces of $\mathcal{G}$ ).

Now consider the inverse sequence

$$
G_{B_{1}}(X) \stackrel{f_{1}}{\leftarrow} G_{B_{1}^{2}}(X) \cup G_{B_{2}^{2}}(X) \stackrel{f_{2}}{\leftarrow \ldots,}
$$

where each bonding map $f_{j}: \bigcup_{i=1}^{j+1} G_{B_{i}^{j+1}}(X) \rightarrow \cup_{i=1}^{j} G_{B_{i}^{j}}(X)$ is defined by

$$
f_{j}=\eta\left(\bigcup_{i=1}^{j} G_{B_{i}^{j}}(X) ; \bigcup_{i=1}^{j+1} G_{B_{i}^{j+1}}(X)\right)
$$

as in §2. Each $f_{j}$ has contractible point-inverses, and is therefore a nearhomeomorphism. By the Approximation Lemma of [6], $\lim _{j \rightarrow \infty} \cup_{i=1}^{j} G_{B_{i}^{\prime}}(X)$ $\approx$ inv $\lim \left(\cup_{i=1}^{j} G_{B_{i}^{j}}(X), f_{j}\right) \approx Q$. Thus the element $A$ has a $Q$-neighborhood in $\mathcal{G}$, and $\mathcal{G} \backslash\{X\}$ is a $Q$-manifold.

It remains to show that $\mathcal{G} \backslash\{X\}$ is properly contractible to infinity. Let $\mathscr{K}$ be a compact subspace of $\mathcal{G} \backslash\{X\}$, and choose $\varepsilon>0$ such that $\rho(K, X)>\varepsilon$ for each $K \in \mathscr{K}$. Let $\mathcal{F}$ be the growth hyperspace $\{F \in \mathcal{G} \mid \rho(F, X) \leqslant \varepsilon\}$. Then the deformation $\left\{\eta_{t}\right\}$ associated with the retraction $\eta(\mathscr{F} ; \mathcal{G}): \mathcal{G} \rightarrow \mathscr{F}$ restricts to the desired proper homotopy of $\mathcal{G} \backslash\{X\}$. Specifically, define $\eta_{t}(F)=\eta\left(F, t s_{F}\right)$, where $s_{F}$ is the smallest value of $s$ for which $\eta(F, s) \in \mathscr{F}$. Each $\eta_{t}$ maps $\mathcal{G} \backslash\{X\}$ into itself, $\eta_{0}=\mathrm{id}, \eta_{1}(\mathcal{G} \backslash\{X\}) \cap \mathscr{K}=\varnothing$, and obviously $\left\{\eta_{t}\right\}$ is proper. Thus $\mathcal{G} \backslash\{X\}$ is a $[0,1)$-stable $Q$-manifold.

4. Inclusion hyperspaces. We first obtain an analogue of Lemma 3.2.

Lemma 4.1. Let $A_{1}, \ldots, A_{n}$ be nonempty closed subsets of a Peano continuum $X$ such that $\cup_{i=1}^{n} A_{i} \neq X$. Then for each $\varepsilon>0$ there exist closed sets $B_{1}=\eta\left(A_{1}, \delta_{1}\right), \ldots, B_{n}=\eta\left(A_{n}, \delta_{n}\right)$, with $0<\delta_{i} \leqslant \varepsilon$ for each $i$, such that the inclusion hyperspace $\cup_{i=1}^{n} 2_{B_{i}}^{X}$ is homeomorphic to $Q$.

Proof. We may assume that $A_{i} \backslash A_{j} \neq \varnothing$, and that in fact $A_{i} \backslash \eta\left(A_{j}, \varepsilon\right) \neq$ $\varnothing$, for each $i \neq j$. Also assume that $\eta\left(\cup_{i=1}^{n} A_{i}, \varepsilon\right) \neq X$. Since $X$ is connected, the proper closed subset $\cup_{i=1}^{n} A_{i}$ has a boundary; reindexing the $A_{i}$, we may suppose that $A_{n} \cap \mathrm{bd}\left(\cup_{i=1}^{n} A_{i}\right) \neq \varnothing$. Then take $B_{n}=\eta\left(A_{n}, \varepsilon\right)$. Note that 
int $B_{n} \backslash \cup_{i=1}^{n-1} A_{i} \neq \varnothing$. By the same argument we may suppose that $A_{n-1} \cap$ $\operatorname{bd}\left(\cup_{i=1}^{n-1} A_{i}\right) \neq \varnothing$. Then take $B_{n-1}=\eta\left(A_{n-1}, \delta\right)$, where $0<\delta<\varepsilon$ is small enough that int $B_{n} \backslash\left(\cup_{i=1}^{n-1} A_{i} \cup B_{n-1}\right) \neq \varnothing$. Note that int $B_{n-1} \backslash \cup_{i=1}^{n-2} A_{i} \neq$ $\varnothing$. Continuing in this fashion we obtain closed sets $B_{1}, \ldots, B_{n}$ such that $\bigcup_{i=1}^{n} B_{i} \neq X$, int $B_{i} \backslash \cup_{j=1}^{i-1} B_{j} \neq \varnothing$ for each $i$, and int $B_{i} \backslash B_{j} \neq \varnothing$ for $i<j$. The proof of Lemma 5.5 of [8] then shows immediately that $\cup_{i=1}^{n} 2_{B_{i}}^{X} \approx Q$.

THEOREM 4.1. If $\mathcal{G}$ is a nontrivial inclusion hyperspace of a Peano continuum $X$, then $\mathcal{G} \backslash\{X\}$ is $a[0,1)$-stable $Q$-manifold.

Proof. One uses the same type of inverse sequence construction as in the proof of Theorem 3.1, with Lemma 4.1 taking the place of Lemma 3.2.

\section{Applications and examples.}

COROLlary 5.1. Let $\mathcal{G}$ be a nontrivial growth hyperspace of a Peano continuum $X$, such that either $X$ contains no free arcs or $\mathcal{G}$ is an inclusion hyperspace. Then the following statements are equivalent:

(1) $\mathcal{G} \approx Q$

(2) $\mathcal{G} \backslash\{X\}$ is contractible;

(3) $\{X\}$ is a $Z$-set in $\mathcal{G}$.

Proof. Obviously (1) $\Rightarrow(2)$, (3). By Chapman's classification theorem [2], every contractible $[0,1)$-stable $Q$-manifold is homeomorphic to $Q \times[0,1)$. Thus $(2) \Rightarrow \mathcal{G} \approx$ Cone $Q \approx Q$. The result of Torunczyk used in the proof of Lemma 3.1 (or an earlier version due to West [16]) shows that (3) $\Rightarrow(1)$.

It was shown in [8] that for $A_{1}, \ldots, A_{n} \in 2^{X}$, the inclusion hyperspace $2^{X}\left(A_{1}, \ldots, A_{n}\right)=\left\{F \in 2^{X} \mid F \cap A_{i} \neq \varnothing\right.$ for each $\left.i\right\}$ is homeomorphic to $Q$, and that the growth hyperspace $C\left(X ; A_{1}, \ldots, A_{n}\right)=C(X) \cap$ $2^{X}\left(A_{1}, \ldots, A_{n}\right)$ is homeomorphic to $Q$ if $X$ contains no free arcs. Alternatively, these results may be deduced from Corollary 5.1. Let $y \in X \backslash$ $\cup_{i=1}^{n}$ bd $A_{i}$. Given $\varepsilon>0$, choose $0<\delta<\varepsilon$ such that $\eta(y, \delta)$ does not intersect $\cup_{i=1}^{n}$ bd $A_{i}$. By Lemma 5.4 of [8], there exists a map $f: 2^{X} \rightarrow 2^{X}$ । $2_{\eta(y, \delta / 2)}^{X}$ such that $\rho(f$, id $)<\delta / 2, f(B) \backslash \eta(y, \delta / 2)=B \backslash \eta(y, \delta / 2)$ for each $B \in 2^{X}$, and if $X$ contains no free arcs, $f$ maps $C(X)$ into itself. It follows that $f$ maps $2^{X}\left(A_{1}, \ldots, A_{n}\right)$ into $2^{X}\left(A_{1}, \ldots, A_{n}\right) \backslash\{X\}$, and if $X$ contains no free arcs, $f$ maps $C\left(X ; A_{1}, \ldots, A_{n}\right)$ into $C\left(X ; A_{1}, \ldots, A_{n}\right) \backslash\{X\}$.

The result (previously unpublished) that $\cup_{i=1}^{n} 2_{A_{i}}^{X}$ is homeomorphic to $Q$ if $\cup_{i=1}^{n} A_{i} \neq X$ also follows from the Corollary 5.1. Let $N$ be any closed subset of $X \backslash \cup_{i=1}^{n} A_{i}$ with nonempty interior; then again by Lemma 5.4 of [8], there exists for each $\varepsilon>0$ a map $f: 2^{X} \rightarrow 2^{X} \backslash 2_{N}^{X}$ such that $\rho(f$, id $)<\varepsilon$ and $f(B) \backslash N=B \backslash N$ for each $B \in 2^{X}$. Thus $f$ maps $\cup_{i=1}^{n} 2_{A_{i}}^{X}$ into $\cup_{i=1}^{n} 2_{A_{i}}^{X} \backslash$ $\{X\}$. If furthermore $X$ contains no free arcs, then $f$ maps $\cup_{i=1}^{n} C_{A_{i}}(X)$ into $\cup_{i=1}^{n} C_{A_{i}}(X) \backslash\{X\}$, and thus $\cup_{i=1}^{n} C_{A_{i}}(X)$ is homeomorphic to $Q$. In this 
case the same argument shows also that both $\bigcup_{i=1}^{n} G_{A_{i}}(X)$ and $\cap_{i=1}^{n} G_{A_{i}}(X)$ are homeomorphic to $Q$.

We can place these results in a more general setting by considering $Q_{0}$-decompositions. Let $Q_{0}=Q \backslash$ point $\approx Q \times[0,1)$. A $Q_{0}$-decomposition of a separable locally compact metric space $Y$ is a star-finite, locally finite closed cover $\left\{\beta_{i}\right\}$ of $Y$ such that each nonempty intersection $\beta_{i_{1}} \cap \cdots \cap \beta_{i_{n}}$, $n \geqslant 1$, is homeomorphic to $Q_{0}$. The nerve $K$ of $\left\{\beta_{i}\right\}$ is the abstract complex whose vertices are the elements of $\left\{\beta_{i}\right\}$ and whose simplices are those subcollections of $\left\{\beta_{i}\right\}$ with a nonempty intersection.

LEMMA 5.1 [5]. Let $\left\{\beta_{i}\right\}$ be a $Q_{0}$-decomposition of $Y$ with nerve $K$. Then $Y \times Q \approx|K| \times Q_{0}$.

Corollary 5.2. Let $X$ be a Peano continuum, with proper closed subsets $A_{1}, \ldots, A_{n}$, and let $K$ be the abstract complex whose vertices are the sets $A_{i}$ and whose simplices are those subcollections of $\left\{A_{i}\right\}$ whose union is a proper subset of $X$. Then $\cup_{i=1}^{n} 2_{A_{i}}^{X}$ is homeomorphic to the cone over $|K| \times Q$. If furthermore $X$ contains no free arcs, then both $\cup_{i=1}^{n} C_{A_{i}}(X)$ and $\cup_{i=1}^{n} G_{A_{i}}(X)$ are homeomorphic to the cone over $|K| \times Q$.

Proof. The collection $\left\{2_{A_{i}}^{X} \backslash\{X\}\right\}$ is a finite $Q_{0}$-decomposition of $\cup_{i=1}^{n} 2_{A_{i}}^{X}$ $\backslash\{X\}$, with nerve $K$. Then since $\cup_{i=1}^{n} 2_{A_{i}}^{X} \backslash\{X\}$ is a $Q$-manifold, $\cup_{i=1}^{n} 2_{A_{i}}^{X} \backslash$ $\{X\} \approx\left(\cup_{i=1}^{n} 2_{A_{i}}^{X} \backslash\{X\}\right) \times Q \approx|K| \times Q_{0}$. Thus $\cup_{i=1}^{n} 2_{A_{i}}^{X}$ is homeomorphic to the one-point compactification of $|K| \times Q \times[0,1)$, i.e., $\cup_{i=1}^{n} 2_{A_{i}}^{X} \approx$ Cone $(|K| \times Q)$. Similarly for $\cup_{i=1}^{n} C_{A_{i}}(X)$ and $\cup_{i=1}^{n} G_{A_{i}}(X)$; in the latter case we use the result established above that $\cap_{i=1}^{k} G_{A_{i}}(X) \approx Q$ if (and only if) $\cup_{i=1}^{k} A_{i} \neq X$.

Note that the cone over $|K| \times Q$ is homeomorphic to $Q$ if and only if $|K|$ is contractible. This includes, but is not limited to, the situation where $\cup_{i=1}^{n} A_{i} \neq X$.

COROLlary 5.3. Let $\mathcal{G}$ be a growth hyperspace of a Peano continuum $X$, such that either $X$ contains no free arcs or $\mathcal{G}$ is an inclusion hyperspace. If for each $\varepsilon>0$ there exists an $\varepsilon$-net $\left\{A_{1}, \ldots, A_{n}\right\}$ in $\mathcal{G}$ whose complex $K$ (in the sense of Corollary 5.2) is contractible, then $\mathcal{G}$ is homeomorphic to $Q$.

Proof. Given $\varepsilon>0$, let $\left\{A_{1}, \ldots, A_{n}\right\}$ be an $\varepsilon / 2$-net in $\mathcal{G}$ with contractible complex $K$. The retraction $\eta=\eta\left(\cup_{i=1}^{n} G_{A_{i}}(X) ; \mathcal{G}\right): \mathcal{G} \rightarrow \cup_{i=1}^{n} G_{A_{i}}(X)$ is within $\varepsilon / 2$ of the identity map. Now suppose $X$ contains no free arcs. Then $\cup_{i=1}^{n} G_{A_{i}}(X)$ is homeomorphic to $Q$, the element $\{X\}$ is a $Z$-set in $\cup_{i=1}^{n} G_{A_{i}}(X)$, and there exists a map $f: \cup_{i=1}^{n} G_{A_{i}}(X) \rightarrow \bigcup_{i=1}^{n} G_{A_{i}}(X) \backslash\{X\}$ such that $\rho(f$, id $)<\varepsilon / 2$. The composition $f \eta: \mathcal{G} \rightarrow \mathcal{G} \backslash\{X\}$ satisfies $\rho(f \eta$, id $)$ $<\varepsilon$, thus $\{X\}$ is a $Z$-set in $\mathcal{G}$, and $\mathcal{G}$ is homeomorphic to $Q$. The analogous 
argument in the case that $\mathcal{G}$ is an inclusion hyperspace uses the retraction $\eta\left(\cup_{i=1}^{n} 2_{A_{i}}^{X} ; \mathcal{G}\right)$.

We also use the $Q_{0}$-decomposition lemma to obtain a converse for Theorems 3.1 and 4.1.

THeORem 5.1. For every $[0,1)$-stable $Q$-manifold $M$ and nondegenerate Peano continuum $X$, there exists an inclusion hyperspace $\mathcal{G}$ of $X$ such that $\mathcal{G} \backslash\{X\} \approx M$.

Proof. By Chapman's triangulation theorem [3], there exists a countable locally finite simplicial complex $K$ such that $|K| \times Q_{0} \approx M$. Let $\left\{\sigma_{i}\right\}$ be an enumeration of the simplices of $K$. Choose a sequence $\left\{U_{i}\right\}$ of disjoint nonempty open sets in $X$ such that $\lim _{i \rightarrow \infty} \bar{U}_{i}=\{p\}$ for some $p \in X$. For each vertex $v$ of $K$, define $U_{v}=\cup\left\{U_{i} \mid v \in \sigma_{i}\right\}$. Then take $\mathcal{G}=\bigcup\left\{2_{X \backslash U_{0}}^{X} \mid v\right.$ a vertex of $K\}$. The cover $\left\{2_{X \backslash U_{0}}^{X} \backslash\{X\}\right\}$ of $\mathcal{G} \backslash\{X\}$ is a $Q_{0}$-decomposition with nerve $K$. Thus $\mathcal{G} \backslash\{X\} \approx(\mathcal{G} \backslash\{X\}) \times Q \approx|K| \times Q_{0} \approx M$.

6. Convex growth hyperspaces. Let $X$ be a convex $n$-cell, and $\operatorname{cc}(X)$ the hyperspace of nonempty closed convex subsets. It is known [11] that $\operatorname{cc}(X)$ is homeomorphic to $Q$ for $n>1$. A nonempty closed subspace $\mathcal{G}$ of $\operatorname{cc}(X)$ is a convex growth hyperspace provided it satisfies the following condition: if $A \in \mathcal{G}$ and $B \in \operatorname{cc}(X)$ such that $B \supset A$, then $B \in \mathcal{G}$.

Let $d$ be the Euclidean metric on a convex $n$-cell $X \subset R^{n}$. Then the contraction $\eta$ of $\operatorname{cc}(X)$ to the point $\{X\}$ is defined as in $\$ 2$, and for convex growth hyperspaces $\mathcal{G} \subset \mathcal{H}$, we also have the strong deformation retraction $\eta(\mathcal{G} ; \mathcal{H})$ of $\mathcal{H}$ to $\mathcal{G}$. Thus every convex growth hyperspace is a retract of $\operatorname{cc}(X)$, and is therefore an $\mathrm{AR}$ and a $Q$-factor.

THEOREM 6.1. If $\mathcal{G}$ is a nontrivial convex growth hyperspace of a convex $n$-cell $X, n>1$, then $\mathcal{G} \backslash\{X\}$ is a $[0,1)$-stable $Q$-manifold.

For the proof of Theorem 6.1 we consider the convex growth hyperspaces $\operatorname{cc}_{A}(X)=\{B \in \operatorname{cc}(X) \mid B \supset A\}$, where $A \in 2^{X}$. We could of course replace $A$ by its convex hull conv $A$. If conv $A \neq X$ and $n>1$, it is also known [11] that $\mathrm{cc}_{A}(X)$ is homeomorphic to $Q$. We first obtain the convex analogue of Lemma 4.1.

Lemma 6.1. Let $A_{1}, \ldots, A_{k}$ be closed subsets of the convex $n$-cell $X, n>1$, such that conv $\left(\cup_{i=1}^{k} A_{i}\right) \neq X$. Then $\cup_{i=1}^{k} \mathrm{cc}_{A_{i}}(X) \approx Q$.

The proof of Lemma 6.1 requires the following technical lemmas.

Lemma 6.2. Let $A \in \operatorname{cc}(X)$ and $p \in \operatorname{bd} X \backslash A$. Then for every $\varepsilon>0$ there exists a hyperplane $H$ strictly separating $A$ and $p$ and such that $\rho\left(X, X \cap H^{+}\right)$ $<\varepsilon$, where $\mathrm{H}^{+}$is the closed half-space of $\mathbf{R}^{n}$ containing $A$. 
Proof. Let $B \subset$ int $X$ be a compact set such that $\rho(X, B)<\varepsilon$. Then $\operatorname{conv}(A \cup B)$ and $p$ are disjoint, and there exists a hyperplane $H$ strictly separating them (Theorem 2.10 of [15]). Clearly, $\rho\left(X, X \cap H^{+}\right)<\rho(X, B)$ $<\varepsilon$.

LEMMA 6.3. Let $A$ be a closed subset of the convex $n$-cell $X, n>1$, such that $A \cap \mathrm{bd} X \neq \varnothing$ and $\operatorname{conv} A \neq X$. Then $A \cap \operatorname{cl}(\mathrm{bd} X \backslash \operatorname{conv} A) \neq \varnothing$.

Proof. Since $X$ is the closed convex hull of the set of its exposed points [15, Theorem 11.6], there exists an exposed point $q$ of $X$ which is not in $\operatorname{conv} A$. Let $H$ be a supporting hyperplane for $X$ through $q$ such that $H \cap X=q$, and let $H^{\prime}$ be the translate of $H$ supporting $A \cap$ bd $X$. Then $H^{\prime}$ must also support $\operatorname{conv} A \cap$ bd $X$, and we have $\varnothing=H^{\prime} \cap A \cap$ bd $X \subset A \cap \operatorname{cl}(b d X \backslash$ $\operatorname{conv} A)$.

Proof of Lemma 6.1. Suppose first that $\bigcup_{i=1}^{k} A_{i} \subset$ int $X$. Let $\mathscr{F}=\{B \in$ $\cup_{i=1}^{k} \operatorname{cc}_{A_{i}}(X) \mid$ int $B \not A_{i}$ for each $\left.i\right\}$. We claim that $\mathcal{F}$ is a $Z$-set in $\cup_{i=1}^{k} \mathrm{cc}_{A_{i}}(X)$ and $\bigcup_{i=1}^{k} \mathrm{cc}_{A_{i}}(X) \backslash \mathscr{F}$ is a $Q$-manifold. The $\varepsilon$-expansion defined by $B \rightarrow \eta(B, \varepsilon)$ gives a map $f: \bigcup_{i=1}^{k} \operatorname{cc}_{A_{i}}(X) \rightarrow \bigcup_{i=1}^{k} \operatorname{cc}_{A_{i}}(X) \backslash \mathscr{F}$ with $\rho(f$, id $)$ $\leqslant \varepsilon$. And for any $B \in \cup_{i=1}^{k} \operatorname{cc}_{A_{i}}(X) \backslash \mathscr{F}$, we have $A_{j} \subset$ int $B$ for some $j$, thus $\operatorname{cc}_{A_{j}}(X)$ is a $Q$-neighborhood of $B$ in $\cup_{i=1}^{k} \operatorname{cc}_{A_{i}}(X)$. Then the theorem of Torunczyk previously cited implies that in this case the $Q$-factor $\cup_{i=1}^{k} \operatorname{cc}_{A_{i}}(X)$ is homeomorphic to $Q$.

Now suppose that $\cup_{i=1}^{k} A_{i} \cap$ bd $X \neq \varnothing$. The proof is by induction on $k$. By Lemma 6.3 we have $\bigcup_{i=1}^{k} A_{i} \cap \operatorname{cl}\left(\operatorname{bd} X \backslash \operatorname{conv}\left(\cup_{i=1}^{k} A_{i}\right)\right) \neq \varnothing$, and we may assume that $A_{k} \cap \operatorname{cl}\left(\mathrm{bd} X \backslash \operatorname{conv}\left(\cup_{i=1}^{k} A_{i}\right)\right) \neq \varnothing$. Let $B_{k}$ be a sufficiently small closed neighborhood of $A_{k}$ in $X$, so that $\operatorname{conv}\left(\cup_{i=1}^{k-1} A_{i} \cup B_{k}\right) \neq X$. We claim that the hyperspace $\cup_{i=1}^{k-1} \operatorname{cc}_{A_{i}}(X) \cup \operatorname{cc}_{B_{k}}(X)$ is homeomorphic to $Q$. By the induction hypothesis $\bigcup_{i=1}^{k-1} \mathrm{cc}_{A_{i}}(X), \operatorname{cc}_{B_{k}}(X)$, and their intersection $\cup_{i=1}^{k-1} \operatorname{cc}_{A_{i} \cup B_{k}}(X)$ are each homeomorphic to $Q$. If we can show that $\bigcup_{i=1}^{k-1} \operatorname{cc}_{A_{i} \cup B_{k}}(X)$ is a $Z$-set in $\bigcup_{i=1}^{k-1} \operatorname{cc}_{A_{i}}(X)$, the claimed result follows from Handel's Sum Theorem for Hilbert cubes [9].

There exists $p \in\left(B_{k} \cap\right.$ bd $\left.X\right) \backslash \operatorname{conv}\left(\cup_{i=1}^{k} A_{i}\right)$. By Lemma 6.2 there exists, for every $\varepsilon>0$, a hyperplane $H$ strictly separating $\operatorname{conv}\left(\cup_{i=1}^{k-1} A_{i}\right)$ and $p$, such that the nearest-point map $\tau: X \rightarrow X \cap H^{+}$satisfies $d(\tau, \mathrm{id}) \leqslant \varepsilon$, where $H^{+}$ is the closed half-space of $R^{n}$ containing $\operatorname{conv}\left(\cup_{i=1}^{k-1} A_{i}\right)$. Then the map $f$ : $\bigcup_{i=1}^{k-1} \operatorname{cc}_{A_{i}}(X) \rightarrow \bigcup_{i=1}^{k-1} \operatorname{cc}_{A_{i}}(X) \backslash \operatorname{cc}_{B_{k}}(X)$, defined by $f(D)=\operatorname{conv} \tau(D)$, satisfies $\rho(f, \mathrm{id}) \leqslant \varepsilon$. Thus $\cup_{i=1}^{k-1} \mathrm{cc}_{A_{i}}(X) \cup \mathrm{cc}_{B_{k}}(X)$ is homeomorphic to $Q$.

We may now routinely obtain the hyperspace $\cup_{i=1}^{k} \mathrm{cc}_{A_{i}}(X)$ as the limit of a monotone inverse sequence of hyperspaces of the above form $\bigcup_{i=1}^{k-1} \operatorname{cc}_{A_{i}}(X)$ $\cup \mathrm{Cc}_{B_{k}}(X)$, by considering successively smaller neighborhoods $B_{k}$ of $A_{k}$, and using as bonding maps the retractions derived from the expansion map $\eta$. This construction is the same as that given in the proof of Theorem 3.1. Thus 
by the inverse sequence approximation lemma, $\cup_{i=1}^{k} c_{A_{i}}(X)$ is homeomorphic to $Q$.

Proof of Theorem 6.1. Let $A \in \mathcal{G} \backslash\{X\}$. There exists a closed neighborhood $\mathcal{R}$ of $A$ in $\mathcal{G}$ such that for each subset $\left\{A_{1}, \ldots, A_{k}\right\} \subset \Re$, $\operatorname{conv}\left(\cup_{i=1}^{k} A_{i}\right) \neq X$. Let $\left\{A_{i}\right\}$ be a dense sequence in $\mathcal{T}$, and consider the inverse sequence

$$
\operatorname{cc}_{A_{1}}(X) \stackrel{f_{1}}{\leftarrow} \operatorname{cc}_{A_{1}}(X) \cup \mathrm{cc}_{A_{2}}(X) \stackrel{f_{2}}{\leftarrow} \cdots,
$$

where each $f_{i}$ is the $\eta$ retraction. By Lemma 6.1 each coordinate space is homeomorphic to $Q$. The limit space is the closed neighborhood $\{F \in$ $\operatorname{cc}(X) \mid F \supset B$ for some $B \in \mathcal{N}\}$ of $A$ in $\mathcal{G}$, and the approximation lemma applies, showing that this neighborhood is homeomorphic to $Q$. The argument that $\mathcal{G} \backslash\{X\}$ is properly contractible to infinity is the same as in the proof of Theorem 3.1.

COROLLARY 6.1. Let $\mathcal{G}$ be a nontrivial convex growth hyperspace of a convex $n$-cell $X, n>1$. Then the following statements are equivalent:

(1) $\mathcal{G} \approx Q$

(2) $\mathcal{G} \backslash\{X\}$ is contractible;

(3) $\{X\}$ is a Z-set in $\mathcal{G}$.

Corollary 6.2. Let $A_{1}, \ldots, A_{k}$ be closed subsets of the convex $n$-cell $X$, $n>1$, with each conv $A_{i} \neq X$, and let $K$ be the abstract complex whose vertices are the sets $A_{i}$ and whose simplices are those subcollections $\left\{A_{i}\right\}$ for which $\operatorname{conv}\left(\cup A_{i}\right) \neq X$. Then $\cup_{i=1}^{k} \operatorname{cc}_{A_{i}}(X)$ is homeomorphic to the cone over $|K| \times$ $Q$.

COROLlaRY 6.3. Let $A$ be a nonempty closed subset of the convex n-cell $X$, $n>1$. Then the convex growth hyperspace $\operatorname{cc}(X ; A)=\{F \in \operatorname{cc}(X) \mid F \cap A \neq$ $\varnothing\}$ is homeomorphic to $Q$, unless $A$ is precisely the vertex set $\left\{v_{1}, \ldots, v_{k}\right\}$ of a polyhedral $X$, in which case $\operatorname{cc}(X ; A)$ is homeomorphic to the cone over $S^{k-2} \times Q$.

Proof. The complex $K\left(x_{1}, \ldots, x_{k}\right)$ associated with a collection $\left\{x_{1}, \ldots, x_{k}\right\}$ of points in $X$, as in Corollary 6.2, is obviously contractible if $\operatorname{conv}\left\{x_{1}, \ldots, x_{k}\right\} \neq X$. On the other hand, if the convex hull of some proper subcollection of $\left\{x_{1}, \ldots, x_{k}\right\}$ is $X$, this subcollection must include all the vertices of $X$; there exists some $x_{i}$ which is not a vertex; the complex $K\left(x_{1}, \ldots, x_{k}\right)$ is star-shaped from $x_{i}$, and therefore contractible. Thus $K\left(x_{1}, \ldots, x_{k}\right)$ is contractible unless $\left\{x_{1}, \ldots, x_{k}\right\}$ is a minimal spanning set for $X$, i.e., the vertex set of $X$, in which case $K\left(x_{1}, \ldots, x_{k}\right)$ is a combinatorial $(k-2)$-sphere.

If $A$ is not the vertex set of $X$, there exists a dense sequence $\left\{x_{i}\right\}$ in $A$ such 
that for each $k,\left\{x_{1}, \ldots, x_{k}\right\}$ is not the vertex set of $X$. Then by the above argument and Corollary 6.2, the hyperspace $\cup_{i=1}^{k} c c_{x_{i}}(X)$ is homeomorphic to $Q$ for each $k$. Since $\lim _{k \rightarrow \infty} \cup{ }_{i=1}^{k} \operatorname{cc}_{x_{i}}(X)=\operatorname{cc}(X ; A)$, consideration of the inverse sequence

$$
\operatorname{cc}_{x_{1}}(X) \stackrel{f_{1}}{\leftarrow} \operatorname{cc}_{x_{1}}(X) \cup \operatorname{cc}_{x_{2}}(X) \stackrel{f_{2}}{\leftarrow} \cdots,
$$

where each $f_{i}$ is the $\eta$ retraction, shows that $\operatorname{cc}(X ; A)$ is homeomorphic to $Q$. The result in the case where $A$ is the vertex set of $X$ follows directly from Corollary 6.2.

As before, Theorem 6.1 has a converse.

Theorem 6.2. For every $[0,1)$-stable $Q$-manifold $M$ and every $n>1$, there exists a convex growth hyperspace $\mathcal{G}$ of a convex $n$-cell $X$ such that $\mathcal{G} \backslash\{X\} \approx$ M.

Proof. First consider the case $n=2$. Let $X$ be a convex 2-cell with a countable set of exposed points $E=\left\{x_{1}, x_{2}, \ldots, x_{\infty}\right\}$, such that $x_{i} \rightarrow x_{\infty}$ and bd $X=\overline{x_{\infty} x_{1}} \cup \overline{x_{1} x_{2}} \cup \overline{x_{2} x_{3}} \cup \cdots$. Let $K$ be a countable locally finite simplicial complex such that $M \approx|K| \times Q_{0}$, and let $\left\{\sigma_{i}\right\}$ be an enumeration of the simplices of $K$. For each vertex $v$ of $K$, set $E_{v}=E \backslash\left\{x_{i} \mid v \in \sigma_{i}\right\}$. Define $\mathcal{G}=\bigcup\left\{\operatorname{cc}_{E_{\mathrm{v}}}(X) \mid v\right.$ a vertex of $\left.K\right\}$. Then $\mathcal{G}$ is a convex growth hyperspace of $X$, and the cover $\left\{\operatorname{cc}_{E_{v}}(X) \backslash\{X\}\right\}$ of $\mathcal{G} \backslash\{X\}$ is a $Q_{0^{-}}$ decomposition with nerve $K$. Thus $\mathcal{G} \backslash\{X\} \approx(\mathcal{G} \backslash\{X\}) \times Q \approx|K| \times Q_{0} \approx$ $M$.

For $n>2$, we consider the convex $n$-cell $X \times I^{n-2}$, and take $\mathcal{G}=$ $\cup\left\{\mathrm{cc}_{E_{\mathrm{r}} \times I^{n-2}}\left(X \times I^{n-2}\right)\right\}$.

A final remark: one can routinely verify (using projections onto finitedimensional convex cells) that all the results of this section remain valid when $X$ is an infinite-dimensional compact convex set in Hilbert space.

\section{REFERENCES}

1. R. H. Bing, Partitioning a set, Bull. Amer. Math. Soc. 55 (1949), 1101-1110.

2. T. A. Chapman, On the structure of Hilbert cube manifolds, Comp. Math. 24 (1972), 329-353.

3.

4. L_ Lectures on Hilbert cube manifolds, CBMS Regional Conf. Ser. in Math., no. 28, Amer. Math. Soc.. Providence, R. I., 1976.

5. D. W. Curtis and G. Kozlowski, Brick decompositions and Q-manifolds, Proc. Amer. Math. Soc. (to appear).

6. D. W. Curtis and R. M. Schori, Hyperspaces of polyheara are Hilbert cubes (submitted).

7. ___ Hyperspaces of Peano continua are Hilbert cubes (submitted).

8. Hyperspaces which characterize simple homotopy type, General Topology and Appl. 6 (1976), 153-165.

9. Michael Handel, On certain sums of Hilbert cubes (preprint).

10. J. L. Kelley, Hyperspaces of a continuum, Trans. Amer. Math. Soc. 52 (1942), 22-36. 
11. Sam B. Nadler, Jr., J. Quinn and Nick M. Stavrakas, Hyperspaces of compact convex sets (preprint).

12. R. M. Schori and J. E. West, The hyperspace of the closed unit interval is a Hilbert cube, Trans. Amer. Math. Soc. 213 (1975), 217-235.

13. __ Hyperspaces of graphs are Hilbert cubes, Pacific J. Math. 53 (1974), 239-251.

14. H. Torunczyk, Concerning locally homotopy negligible sets and characterization of $l_{2}$-manifolds, Fund. Math. (to appear).

15. F. A. Valentine, Convex sets, McGraw-Hill, New York, 1964.

16. J. E. West, The subcontinua of a dendron form a Hilbert cube factor, Proc. Amer. Math. Soc. 36 (1972), 603-608.

17. M. Wojdyslawski, Retractes absolus et hyperespaces des continus, Fund. Math. 32 (1939), 184-192.

18. Raymond Y. T. Wong, Non-compact Hilbert cube manifolds (preprint).

Department of Mathematics, louisiana State University, Baton Rouge, Louisiana 70803 